\title{
A Short Note on Dietary Components and Noncommunicable Diseases
}

\section{Taskina M, Mohammed FAS, Pronay AMTA and Rashed N*}

Microbiology, Department of Life Sciences (DLS), School of Environment and Life Sciences (SELS), Independent University, Bangladesh (IUB), Bangladesh

*Corresponding author: Rashed Noor, Associate Professor (Microbiology), Department of Life Sciences (DLS), School of Environment and Life Sciences (SELS), Independent University, Bangladesh (IUB), Plot 16, Block B, Aftabuddin Ahmed Road, Bashundhara, Dhaka 1229, Bangladesh, Tel: +8801749401451; Email: rashednoor@iub.edu.bd

\section{Mini Review}

Volume 5 Issue 4

Received Date: November 09, 2020

Published Date: November 20, 2020

DOI: $10.23880 /$ oajmb-16000177

\section{Abstract}

Human nutrition and associated metabolism together with the development of non-communicable diseases are the key factors for the mass public health maintenance around the world. The dietary habits; i.e., the optimal consumption of the dietary factors, evaluation of the etiologic effects of the nutrients on the metabolic aftermaths, and assessment of the risk factors of different diets on the disease progression are hence major health legislative attributes. Current review shortly explained the impact of diets on public health sustainability.

Keywords: Nutrition; Diet; Diseases

Abbreviations: NCDs: Noncommunicable Diseases; CVD: Cardiovascular Disease; SSBs: Sugar-Sweetened Beverages; CHD: Coronary Heart Disease; LDL: Low-Density Lipoprotein; BMI: Body Mass Index; BAZ: BMI for Age Z-Score; DBM: Double Burden of Malnutrition.

\section{Background: Dietary Intake and Onset of Diseases}

Health properties of different nutrients within food items largely depend on the critical inter-relation between the ingested foods according to the dietary configurations which in turn impart a significant impact on the health improvement as well as hindering the onset of diseases. Such dietary strategies (diet models or the food guidance tools) aid to select foods for consumption in consideration of delivering the optimal nutrient intake with a concomitant possibility of lowering the risk of noncommunicable diseases (NCDs) like coronary heart disease, stroke, and diabetes $[1,2]$. According to the nutritional epidemiologic investigations, nutrient deficiencies are very much likely to result from the insufficient intake or absorption of specific nutrients from different food groups, which ultimately accounts for the development of certain diseases like the cardiovascular disease (CVD), onset of cancer, and most commonly, protruding diabetes [1]. Unlike the nutrient deficiency, the carbohydrate rich diet (with added sugar) and even with a lower consumption of bad fat may instigate cardiometabolic disease risk accompanied with type 2 diabetes while the higher dietary saturated fat may lead to the CVD [1,3]. Indeed, the least processed, bioactive-rich fruits, vegetables, nuts, seeds, beans, legumes, and whole grains have been reported to impart the protective effects against the NCDs, whereas the highly processed meats and the sugar-sweetened beverages (SSBs) have been noticed with the instigative potential to trigger the coronary heart disease (CHD), stroke, and diabetes [2,4]. Lots of published literatures are available now which really heighten the general awareness about the appropriate dietary regulations. Indeed, physiological and metabolic uunderstanding of the 


\section{Open Access Journal of Microbiology \& Biotechnology}

role of carbohydrate, proteins, fats and sterols, vitamins and minerals in human nutrition is of great significance for the maintenance of health and prevention of disease.

\section{Irregular Dietary Consumption Effect}

Both synergistic and/or antagonistic interactions have been reported within the nutrients and the dietary patterns as can be exemplified by the increased absorption of nonheme iron in presence of vitamin $C$, while a competitive inhibition of zinc absorption has been noticed through iron $[1,5]$. On the contrary, the high cholesterol rich eggs which are also plentiful in amino acids and several micronutrients, have been noticed to reduce the risk of CVD [1,5]. Besides, it should be noted that fish and seafoods as well as the longchain omega-3s has been reported to lower the risk of CHD; and yogurt, which consists of active probiotics, have been found to lower the risk of diabetes [2]. Another example antagonistic interaction is the development of undesirable effects on the disease state upon supplementation of specific amino acids or cofactors which are actually supposed to impart beneficial responses [6]. Glutamine supplementation in the diets of cancer patients with cancer may promote accelerated tumor growth although this amino acid is important as nutrient sources for many cells. The supplementation of arginine has been noticed to trigger nitric oxide synthesis as well [6]. Another risk factor is the overnutrition (due to the excessive accumulation of fat in the adipose tissue) during the childhood through the intake of high fats, sugars, energy-dense foods, which in turn results in the NCDs like obesity, diabetes, hypertension, asthma, coronary artery disease and cancer [7].

Indeed, the long-term increased consumption of a high fructose diet together with the dietary fats lead to the weight gain, and obesity; and besides, it is to be pondered that the increase of childhood obesity may trigger the onset of type 2 diabetes in the adult stage; and also, may instigate the risk of atherosclerosis [6]. To achieve the benefits from dietary intake, along with the planned dietary schedule, intake of adequate fiber may reduce the low-density lipoprotein (LDL) levels, C-reactive protein levels, apolipoprotein levels, and blood pressure; and thereby lower the risk of CVD and coronary heart disease (CHD) [6]. Certain parameters like the body mass index (BMI), BAZ (BMI for age Z-score), double burden of malnutrition (DBM) need to be in consideration in order to monitor the probability of the occurrence of such diet induced NCDs.

\section{Conclusion}

In fine, the study of the relationship of dietary nutrition and the maintenance of health is required in order to prevent the non-communicable diseases. The impact of sufficient nutrition resulting from accurate dietary plan is thus significant in terms of weight control and the development of chronic disease as well as the expression of the important genes for the appropriate absorption and metabolism of the foods. In order to ensure the sound level of mass public health as well as to get rid of child malnutrition, the knowledge of human nutrition, nutrient metabolism and the associated implications of the earned knowledge is required for the improved clinical outcomes.

\section{References}

1. Tapsell LC, Neale EP, Satija A, Hu FB (2016) Foods, Nutrients, and Dietary Patterns: Interconnections and Implications for Dietary Guidelines. Adv Nutr 7(3): 445454.

2. Micha R, Shulkin ML, Peñalvo JL, Khatibzadeh S, Singh GM, et al. (2017) Etiologic effects and optimal intakes of foods and nutrients for risk of cardiovascular diseases and diabetes: Systematic reviews and meta-analyses from the Nutrition and Chronic Diseases Expert Group (NutriCoDE). PLoS One 12(4): e0175149.

3. Malik VS, Popkin BM, Bray GA, Després JP, Willett WC, et al. (2010) Sugar-sweetened beverages and risk of metabolic syndrome and type 2 diabetes: a metaanalysis. Diabetes Care 33(11): 2477-2483.

4. Sandström B (2001) Micronutrient interactions: effects on absorption and bioavailability. Br J Nutr 85 (S2): S181-S185.

5. Rong Y, Chen L, Zhu T, Song Y, Yu M, et al. (2013) Egg consumption and risk of coronary heart disease and stroke: dose-response meta-analysis of prospective cohort studies. BMJ 346: e8539.

6. Ross AC, Caballero BH, Cousins RJ, Tucker KL, Ziegler TR (2012) Modern nutrition in health and disease: Eleventh edition. Wolters Kluwer Health Adis (ESP), pp: 1616.

7. Ali MS, Kassahun CW, Wubneh CA (2020) Overnutrition and Associated Factors: A Comparative Cross-Sectional Study between Government and Private Primary School Students in Gondar Town, Northwest Ethiopia. J Nutr Metab 2020: 3670895. 